\title{
Specific speed-based pump flow rate estimator for large-scale and long-term energy efficiency auditing
}

\author{
Santeri Pöyhönen • Tero Ahonen • Jero Ahola • \\ Pekka Punnonen • Simo Hammo • Lauri Nygren
}

Received: 19 April 2016 / Accepted: 13 November 2018/Published online: 27 November 2018

(C) The Author(s) 2018

\begin{abstract}
Due to their notable share of the total energy consumption of industries, pumps hold particular significance in industrial energy audits. In industrial facilities, pumps exist in large numbers, which leads to only the pumps most critical to the process being monitored. Meanwhile, many old or poorly sized pumps may operate inefficiently without attention. To determine the energy efficiency of a pump, information on the pump's flow rate is required. To this end, flow meters are often used to determine the flow rate produced by a pump, which can be used in determining the pump's specific energy consumption. However, flow meters tend to be costly and impractical for some applications and are laborious for energy auditing, if no preexisting metering can be found in the audited process setup. Thus, soft sensor approaches to estimate flow rate are suggested by several authors. However, the use of model-based flow rate estimators is still uncommon, because they require detailed information on the pump characteristic curves and manual configuration of the device employing the flow rate estimator. This paper introduces a novel flow rate estimator tuned with only four variables: the nominal flow rate, head, shaft power and rotational speed of a centrifugal pump. The estimator operation is based on
\end{abstract}

S. Pöyhönen $(\bowtie) \cdot$ T. Ahonen $\cdot$ J. Ahola $\cdot$ P. Punnonen

S. Hammo

Lappeenranta University of Technology, School of Energy

Systems, P.O. Box 20, 53851 Lappeenranta, Finland

e-mail: santeri.poyhonen@lut.fi

L. Nygren

ABB Oy Marine \& Ports, Helsinki, Finland the expected characteristic performance curves of the centrifugal pump according to its specific speed. Due to a small number of required input parameters, and no need for manufacturer's pump curves, the proposed estimator is easier to commission than existing solutions available both in fixed speed systems and commercial variable-speed drives. The performance and accuracy of the presented estimator are studied by comparing it with the basic $Q P$ and $Q H$-curve-based estimation methods through simulations with four different centrifugal pumps and through measurements with two different centrifugal pumps in a test laboratory. In the experimental evaluation, the suggested estimator estimated the flow rate produced by centrifugal pump with an error of $7-15 \%$ of the nominal flow rate through a flow rate range of $60-100 \%$ of the nominal flow rate. Thus, the presented method is accurate enough to be used in continuous large-scale energy auditing, for example to highlight inefficiently running pumps from large pump populations.

Keywords Centrifugal pump · Estimation - Flow rate . Soft sensing $\cdot$ Large-scale energy auditing

\section{Introduction}

Pumps account for approximately $8 \%$ of the global electric energy consumption (Waide and Brunner 2011). The role of energy efficiency in securing competitiveness, security and sustainability of energy systems has been widely recognized, (European 
Commission 2014) and legislative measures have been taken to improve the energy efficiency within the European Union (European Commission 2012). Legislative pressure to enhance energy efficiency and discovered energy saving potential in electric motor systems (de Almeida et al. 2015; United States Department of Energy 1998; Fleiter and Eichhammer 2012; Kaya et al. 2008) work as incentives to conduct energy audits in the industrial sector (Saidur 2010; Price and Lu 2011). An energy audit provides situational information on the energy consumption and energy-saving possibilities of an energy consumer (Backlund and Thollander 2015).

Flow rate produced by a centrifugal pump is often essential information for the control and monitoring of fluid-related processes as well as for determining the pump's energy efficiency as a part of an energy audit (Mamade et al. 2015). Information on flow rate is typically generated with sensors dedicated for this purpose, such as electromagnetic, orifice plate or ultrasonic flow meters (Nesbitt 2006). However, they can be costly, prone to failure, require maintenance and may simply be an impractical option for processes, where the fluid level or pressure is the primary controlled variable. In some cases, it may be hard to find a suitable operating location for a flow meter in a pumping system. In addition, using flow meters to define the output of a pump for energy auditing purposes requires manual labour if there is no preexisting metering in the process setup. Thus, energy audits are often only conducted on the most critical pumps of processes, which may leave large populations of less important pumps running inefficiently. To this end, soft sensor approach can be a viable solution for getting information on the produced flow rate without additional hardware or sensors. In soft sensing, the flow rate is determined with the help of a model-based estimator that is tuned with the known characteristics of the pump or the surrounding process.

Several soft-sensing methods have been developed, with which a pump's operating state can be estimated without direct metering of the pump's output. The methods are based on either the flow rate vs. head $(Q H)$ or flow rate vs. shaft power curve $(Q P)$ of a pump, or both (Leonow and Mönnigmann 2013; Ahonen et al. 2012a; Tamminen et al. 2014). The operating point of a pump on its characteristic curve can be located by defining the produced head with pressure measurements across the pump and applying it to its $Q H$ curve, or by estimating or measuring the shaft power of the pump and applying it to the pump's $Q P$ curve. Using a $Q P$ - curve-based method, the shaft power can be estimated through motor models as in (Ahonen et al. 2012b; Tinazzi and Zigliotto 2015), with a VSD (Nash 1997), or it can be measured using, for instance, shaft torque and rotational speed sensors. As the estimation accuracy of this method is directly affected by the shape of the characteristic $Q P$ curve (i.e. $\mathrm{d} P / \mathrm{d} Q$ ), the method is usually only applied to radial-flow devices. For $Q H$ curve-based flow rate estimation, pressure measurements present in the piping near a pump can be used to define the produced head.

Electrical current measurements can be used in an electric motor model to estimate the shaft power supplied to a pump and to enable the use of a flow rate estimation method, as demonstrated in (Ahonen et al. 2012b). Electrical current measurements exist in most pump systems for condition monitoring purposes. In addition, load monitors and some soft starters, which can be found in many pump systems, enable continuous monitoring of the electrical current.

Furthermore, a possible unit for employing these estimators is the variable-speed drive (VSD), which is increasingly often employed in pump systems. Modern VSDs are capable of providing estimates for the shaft torque, rotational speed and power consumption of a flow device. The accuracy of these estimates has been found to be sufficient to be used in verifying whether a pump runs in the vicinity of its best efficiency point (BEP) (Ahonen et al. 2011; Ahonen et al. 2010).

Some commercially available VSDs utilize the $Q P$ curve of a pump as a model for the pump's operation at its nominal speed. The use of the basic $Q P$ curve estimation method in a VSD often requires manual configuration of over ten parameters, making the use of flow calculation a too laborious task for the end user. Therefore, simplified flow rate estimators are an interesting option to be implemented in a VSD, if the smaller amount of variables does not compromise their estimation accuracy. Such a solution is already available in the ITT PS200 VSD, where the $Q P$-curve-based estimation method is tuned with four variables: power consumption at zero flow, flow rate and power consumption at the pump best efficiency point (BEP) and the rotational speed at which these variables are known (Kernan et al. 2007).

By using a data collecting and storing system together with the information gained from an estimation method, it can be possible to remotely monitor the operation of pumps. Thus, with an Internet connection and an 
easy-to-employ pump monitoring method, a large population of pumps can be continuously monitored with a relatively low cost. A monitoring method, which can be utilized with little effort and which can cover a large amount of pumps could significantly benefit pump system operators and maintenance staff carrying out activities such as mass auditing and condition monitoring.

In this paper, a novel approach to implement a flow rate estimator is presented. The estimator is based on the expected dependence of the pump's $Q P$ and $Q H$ curves based on the pump specific speed $n_{\mathrm{q}}$. The presented estimator uses a continuous measurement or estimate of the pump shaft power, or a measurement of the head produced by the pump as an input and continuously produces an estimate for the pump flow rate as an output. Also, this flow rate estimator can be tuned with only four variables (BEP values for flow rate, head and power consumption of the pump and the rotational speed at which these variables are known). Unlike ITT's Smartflow method included in their VSD PS220, which requires running the pump against a closed discharge valve to define some of the tuning variables, tuning of the presented estimator is possible with the nominal flow rate, head, shaft power and rotational speed of the pump. However, the model's simplicity in terms of small number of parameters and the assumptions made may compromise the estimation accuracy. This is studied by comparing the proposed estimator with the basic $Q P$ and $Q H$-curve-based estimation methods through simulations with four different centrifugal pumps and through measurements with two different centrifugal pumps in a test laboratory.

The study is started by describing the operating principle of the proposed flow rate estimator in "Flow rate estimation with specific speed-based estimator". Simulation and experimental evaluations of the proposed estimator are provided in "Analysis of estimator operation through simulations and laboratory evaluation". Finally, the obtained results are summarized with conclusions given in "Conclusion".

\section{Flow rate estimation with specific speed-based estimator}

Through a literature review, it was found that works exist, in which universal dimensionless characteristic curves for centrifugal pumps are presented. Gülich (Gülich 2014) and Stepanoff (Stepanoff 1957) have published such relative dimensionless characteristic curves for head, efficiency and shaft power for a range of specific speeds. Specific speed $n_{\mathrm{q}}$ characterizes the pump impeller shape regardless of pump size and can be defined on the basis of the pump's BEP values with the equation

$n_{\mathrm{q}}=n_{\mathrm{BEP}} \frac{\sqrt{Q_{\mathrm{BEP}}}}{H_{\mathrm{BEP}}^{3 / 4}}$,

where $n$ is the rotational speed (rpm), $Q$ is flow rate $\left(\mathrm{m}^{3} / \mathrm{s}\right)$ and $H$ is the head of the pump $(\mathrm{m})$. The BEP values required to calculate the specific speed of a pump can be found in the files received along with the pump upon purchase, in the pump user's device database or, in some cases, on the pump nameplate. ${ }^{1}$ The characteristic $Q P$ and $Q H$ curves of the pump in question can then be estimated on the basis of the calculated specific speed and the dimensionless characteristic curves. Furthermore, the estimated curves can be used in a pump model to estimate the flow rate produced by a pump. To this end, the relative flow rate vs. shaft power and flow rate vs. head curves presented in (Gülich 2014; Stepanoff 1957) were digitized and polynomial curves were fitted on the digitized data. To determine a sufficient order for the polynomial equations, an analysis was carried out, where the goodness of fit was tested with polynomials of degrees of 3, 4 and 5. The norm of residuals and coefficient of determination were calculated for each of the polynomial curves fitted on the digitized Stepanoff's set of $Q P$ curves. The average of the norm of residuals of all the curves and the corresponding average coefficient of determination $\left(R^{2}\right)$ are shown in Table 1 . The average of the norm of residuals for specifically the $n_{\mathrm{q}}=17-77$ curves is also shown in the table, because the curves of $n_{\mathrm{q}}>77$ would not be suitable for flow rate estimation due to their low $|\mathrm{d} P / \mathrm{d} Q|$ and because the present study focuses on centrifugal pumps, whereas $n_{\mathrm{q}}>77$ encompasses mostly mixed flow and axial pumps.

Considering the accuracy of the reference data, no significant improvement in the goodness of fit can be achieved by increasing the polynomial degree from 3 to higher. Thus, third degree polynomials were fitted on

\footnotetext{
${ }^{1}$ In most cases, the pump nameplate includes the duty point of the pump, which can significantly differ from the pump's BEP point.
} 
Table 1 The goodness of fit tested with polynomial degrees of 3 , 4 and 5

\begin{tabular}{llll}
$\begin{array}{l}\text { Degree } \\
\text { of residuals }\end{array}$ & $\begin{array}{l}\text { Average norm of residuals, } \\
\text { when } n_{\mathrm{q}}=17-77\end{array}$ & $\begin{array}{l}\text { Average } \\
R^{2}\end{array}$ \\
\hline 3 & 13.935 & 6.723 & 0.9983 \\
4 & 8.721 & 4.141 & 0.9993 \\
5 & 5.892 & 3.485 & 0.9996 \\
\hline
\end{tabular}

each of the digitized curves. The digitized curves and the third degree polynomials fitted on them are shown in Fig. 1. In the graphs, solid lines represent digitized values and dashed lines represent the third degree polynomial curves fitted on the corresponding digitized data.

When the $n_{\mathrm{q}}$ of a given pump is between the values of $n_{\mathrm{q}}$ for which Gülich (Gülich 2014) and Stepanoff (Stepanoff 1957) have provided characteristic pump curves, the relative characteristic $Q P$ and $Q H$ curves of the pump at its nominal rotational speed can be determined by interpolating the coefficients of the pump's relative curve's polynomial equation based on the surrounding curves' equations. Once the relative $Q P$ and $Q H$ curves for the pump have been generated, a measurement or an estimate for the shaft power or a measurement of the pump's head can be used to derive an estimate for the flow rate produced by the pump.

As seen in Fig. 1, it should be noted that depending on specific speed, either the $Q H$ - or $Q P$-based estimation approach may prove more feasible than the other. At the lower end of the $n_{\mathrm{q}}$ values of the range for centrifugal pumps (approximately $n_{\mathrm{q}}=20-50$ ), the accuracy of $Q P$-curve-based flow rate estimation will be at its highest, because $|\mathrm{d} P / \mathrm{d} Q|$ can be seen to decrease with increasing specific speed. The opposite effect can be observed in the relative $Q H$ curves, where increasing specific speed leads to a greater $|\mathrm{d} H / \mathrm{d} Q|$. The aim of this study is to evaluate the performance of both approaches individually, and if both shaft power and head measurements are available, the uncertainty calculation method presented in (Tamminen et al. 2014) can, for instance, be employed to determine which approach is more likely to produce accurate results with a certain pump.

If the pump is variable-speed-driven, the rotational speed estimate provided by a VSD and the affinity laws for the flow rate, head and required shaft power can be used to determine the $Q P$ and $Q H$ curves at rotational speeds that differ from $n_{\mathrm{BEP}}$
(Gülich 2014; Sulzer Pumps Ltd., Centrifugal pump handbook 1998; Volk 2005):

$$
\begin{aligned}
& \frac{Q}{Q_{\mathrm{BEP}}}=\frac{n}{n_{\mathrm{BEP}}} \\
& \frac{H}{H_{\mathrm{BEP}}}=\left(\frac{n}{n_{\mathrm{BEP}}}\right)^{2}
\end{aligned}
$$

$\frac{P}{P_{\mathrm{BEP}}}=\left(\frac{n}{n_{\mathrm{BEP}}}\right)^{3}$

The coefficients of the third-degree polynomial equations for the curves fitted on the digitized sets of curves are presented in Table 3 of Appendix. To calculate the relative shaft power, head or flow rate, the following equations may be used:

$$
\begin{aligned}
& P^{*}=c_{1}\left(n_{\mathrm{q}}\right) Q^{* 3}+c_{2}\left(n_{\mathrm{q}}\right) Q^{* 2}+c_{3}\left(n_{\mathrm{q}}\right) Q^{*}+c_{4}\left(n_{\mathrm{q}}\right) \\
& H^{*}=c_{1}\left(n_{\mathrm{q}}\right) Q^{* 3}+c_{2}\left(n_{\mathrm{q}}\right) Q^{* 2}+c_{3}\left(n_{\mathrm{q}}\right) Q^{*}+c_{4}\left(n_{\mathrm{q}}\right) .
\end{aligned}
$$

In the equations above, (2)-(4) may be applied when necessary, to convert the equations to non-BEP rotational speeds. A flow diagram of the presented method is illustrated in Fig. 2.

It is worth noting that the curves provided by Stepanoff for specific speeds $n_{\mathrm{q}}=17-110$ are mentioned to apply to double-suction pumps. In contrast, the pumps, with which the performance of the presented flow rate estimator is analysed in this paper, are of the single-suction type. However, in this study, the curves are used as such, since the $n_{\mathrm{q}}$ of both single-suction and double-suction pumps are calculated based on their output values.

In addition to the curves by Gülich and Stepanoff, no other curves were found in the literature review conducted for the present study. The considered curve sets differ from each other, which is especially visible in the relative shaft power and head values they indicate at zero flow rate at $n_{\mathrm{q}}>80$. At lower specific speeds, however, the difference is not as drastic, and comparing the curve sets as bases for the flow rate estimator is reasonable. 
Fig. 1 Relative $Q P$ and $Q H$ characteristic curves provided for a range of pump specific speeds. Here, $P^{*}=P / P_{\mathrm{BEP}}, H^{*}=H / H_{\mathrm{BEP}}$ and $Q^{*}=Q / Q_{\mathrm{BEP}}($ Gülich 2014; Stepanoff 1957)
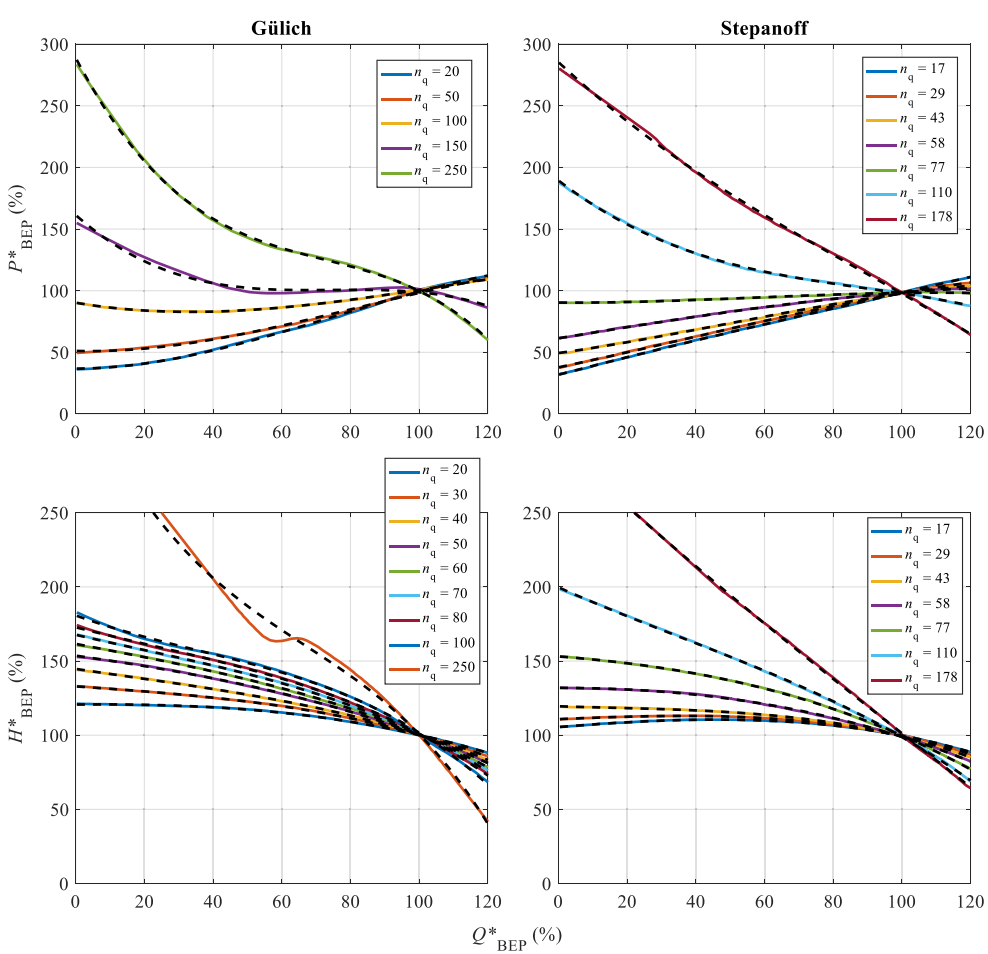

The accuracy of the presented method can suffer because of phenomena that alter the performance of a pump during its lifetime, such as impeller wear or accumulation of matter inside the pump. This effect, however, is also present in the already existing estimation methods, in which manufacturers' $Q P$ and $Q H$ curves are used as pump models. Furthermore, the difference between the curve sets by Gülich and Stepanoff hints at the fact that pumps of equal specific speeds may have curves of different shapes, especially pumps that have been designed several decades apart. The generalization that stems from using a single set of curves can be expected to limit the accuracy of the presented method. However, the aforementioned uncertainty could be reduced by utilizing more modern corresponding curves when the operation of modern pumps is estimated. Such curve sets could, for example, be compiled from pump curves of certain pump manufacturers to represent their pumps or certain pump types of theirs.
Fig. 2 The principle of the presented flow rate estimation method

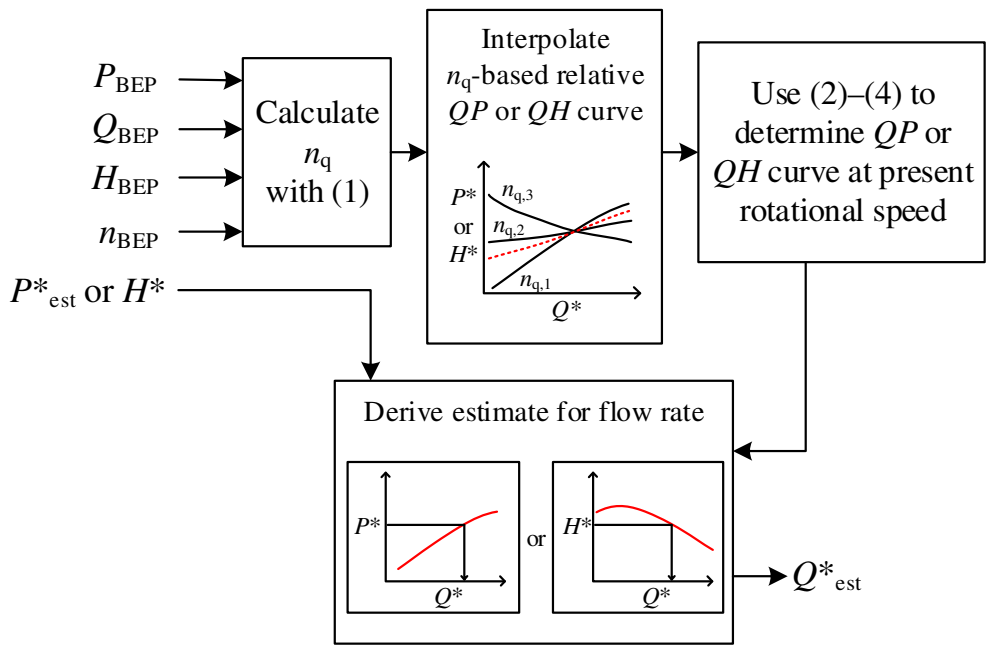




\section{Analysis of estimator operation through simulations and laboratory evaluation}

Using the method described in the previous chapter, the relative $Q P$ and $Q H$ curves of four pumps with different specific speeds were generated with linear interpolation based on the curves published by Gülich and Stepanoff (Gülich 2014; Stepanoff 1957). Centrifugal pumps used in industries typically fall within the range of specific speeds of $n_{\mathrm{q}}=20-50$. In addition to the two Sulzer Ahlstar series pumps present in the pumping laboratory system used in the practical evaluation of the presented estimator, two pumps from the other end of the aforementioned specific speed range were chosen for the analysis. The pumps chosen for the analysis are shown in Table 2.

\section{Laboratory test setup}

To evaluate the accuracy of the presented flow rate estimation method, the actual $Q P$ and $Q H$ curves of Sulzer Ahlstar pumps A11-50 and A22-80 were determined with a laboratory test setup. In addition to the two tested pumps, the laboratory pumping system includes piping, a water tank, through which water is taken, a control valve and metering. The pumps were run at constant rotational speeds of $1420 \mathrm{rpm}$ for A11-50 and $1450 \mathrm{rpm}$ for A2280 , and the flow was throttled with a control valve to record a range of operating points, upon which the characteristic $Q P$ and $Q H$ curves could be fitted. The laboratory measurement setup is illustrated in Fig. 3.

To determine the operating points on the $Q P$ and $Q H$ curves, the shaft power, head and produced flow rate of the pumps were defined and recorded. Shaft power was calculated with

$P_{\text {shaft }}=T \cdot \omega=T \cdot \frac{2 \cdot \pi \cdot n}{60}$, where $T$ is torque (Nm), $\omega$ is angular speed $(\mathrm{rad} / \mathrm{s})$ and $n$ is rotational speed (rpm). For A11-50, shaft power was calculated using torque and rotational speed estimates provided by the frequency converter ABB ACS880, which was used to run the pump. For A22-80, shaft power and rotational speed were measured with a Dataflex 22/100 torque and speed measurement shaft. Head was measured with pressure transducers, and flow rate was measured with ABB's FEP321 electromagnetic flow meter. The measurement data was recorded with a measurement computer running the National Instruments software LabVIEW.

A third-degree polynomial curve was fitted on the measured operating points to represent the pump's characteristic $Q P$ and $Q H$ curves. Then, the published BEP values for shaft power, head and flow rate were applied to convert the curve into its dimensionless relative form. The coefficients of the measured curves' equations are presented in Tables 3 and 4 of Appendix.

\section{$Q P$-curve-based flow rate estimation}

The relative shaft power vs. flow rate curves of the four studied pumps, both generated by the presented method and as provided by the manufacturers, along with the measured curves, are shown in Fig. 4. In the case of the lower $n_{\mathrm{q}}$ pumps, for which measurements were conducted, the practical accuracy of the presented estimation method can now be evaluated and compared with the accuracy of the basic $Q P$ method, which is based on the manufacturer's $Q P$ curve. For the higher $n_{\mathrm{q}}$ pumps, as no measured curves are available, the presented method is evaluated with respect to the manufacturer's $Q P$ curve.

In Fig. 4, it can be seen that the curves generated using Stepanoff's set of $n_{\mathrm{q}}$-based relative characteristic curves better comply with the manufacturer's curves than those based on Gülich's corresponding set of

Table 2 The pumps, for which the published and method-based characteristic curves were compared (Apex Fluid Engineering Ltd, "TD End-Suction Pumps 2003; Sulzer Pumps Finland Oy n.d.; Sulzer Pumps Finland Oy 2005)

\begin{tabular}{lllllll}
\hline Pump & $n_{\mathrm{q}}$ & $H_{\mathrm{BEP}}(\mathrm{m})$ & $Q_{\mathrm{BEP}}\left(\mathrm{m}^{3} / \mathrm{s}\right)$ & $P_{\mathrm{BEP}}(\mathrm{kW})$ & $n_{\mathrm{BEP}}(\mathrm{rpm})$ & Impeller diameter (mm) \\
\hline Sulzer Ahlstar A11-50 & 24 & 13.6 & 0.014 & 2.7 & 1420 & 210 \\
Sulzer Ahlstar A22-80 & 28 & 21.0 & 0.037 & 9.8 & 1450 & 265 \\
Apex TD 200-315 & 51 & 24.6 & 0.149 & 44.3 & 1475 & 307 \\
Apex TD 65-125 & 52 & 18.5 & 0.0254 & 6.2 & 2900 & 131 \\
\hline
\end{tabular}



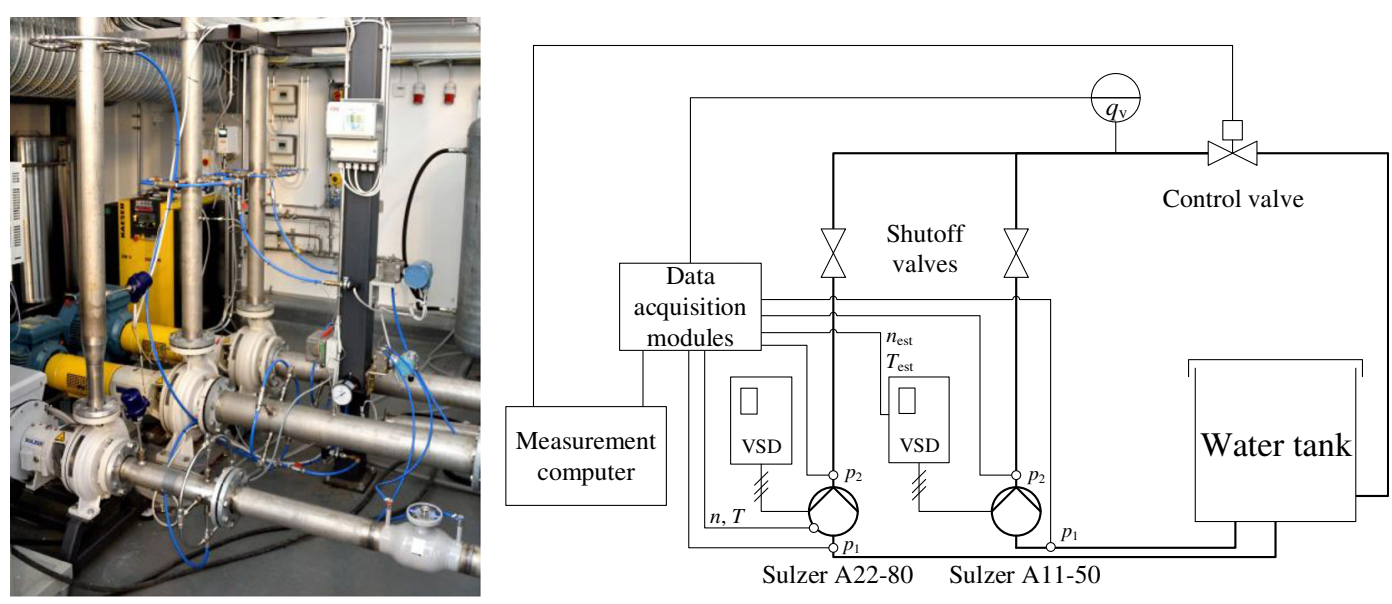

Fig. 3 The laboratory measurement setup

curves. Using Gülich's work as the source for the $n_{\mathrm{q}}$ based relative characteristic curves, the difference between the flow rate derived from the manufacturer's curve and the curve generated with the presented method is significant at low relative flow rates.

It can also be seen that two pumps of a similar specific speed but of two different sizes in terms of nominal shaft power can have relative $Q P$ curves of different shapes. More specifically, the published manufacturer's curve for APEX TD 65-125 has a greater $\mathrm{d} P * / \mathrm{d} Q *$ than that of the curve for APEX TD 200-315. The presented method, as suggested, does not take into account the possibility that pumps of different sizes in terms of nominal shaft power have relative $Q P$ curves of different shapes.

The measured $Q P$ curves can be seen to noticeably differ from the manufacturer's $Q P$ curve and the curves generated with the presented estimation method. For both pumps, the shut-off power indicated by the manufacturer's curve is higher than the actual measured one. For Sulzer Ahlstar A11-50, the difference between the measured and published curves is almost static, whereas for Sulzer Ahlstar A22-80, it is dynamic and the curves intersect at approximately $20 \%$ of the BEP flow rate.

The measured estimation errors of the analysed curves are presented in Fig. 5. The error is calculated as follows: a relative flow rate is considered, then the corresponding shaft power according to the measured relative $Q P$ curve is determined, and finally the acquired relative shaft power is applied to the published and $n_{\mathrm{q}^{-}}$ based curves to determine the flow rate according to them. The error is defined as the difference between the initially considered flow rate and the flow rate derived in the aforementioned way. For Sulzer Ahlstar A11-50, the analysed estimation methods tend to underestimate the produced flow rate. In contrast, for Sulzer Ahlstar A2280 , the estimation methods provide flow rate estimates, which are greater than the actual measured flow rate. The results suggest that the presented estimation method can produce flow rate estimates close to the basic $Q P$ curve-based method in terms of accuracy when Stepanoff's work is used as the source for the $n_{\mathrm{q}}$-based relative characteristic curves. The difference between the flow rate estimate of the presented method and the measured flow rate is 7 to $15 \%$ of $Q_{\mathrm{BEP}}$ through a flow rate range of 60 to $100 \%$ of $Q_{\mathrm{BEP}}$. At $120 \%$ of $Q_{\mathrm{BEP}}$ for Sulzer A22-80, the error is $23 \%$, which is caused by the Stepanoff-based curve's third-degree polynomial shape and location. With this accuracy, the presented method will be able to determine whether a pump is operating outside its preferred operating region. Thus, instead of laborious and costly metering and instrumentation, the presented flow rate estimator can be used in large-scale energy auditing to spot inefficiently running pumps from large pump populations.

In Fig. 6, differences of the manufacturers' $Q P$ curves and the curves generated with the presented $n_{\mathrm{q}}{ }^{-}$ based method are illustrated. The difference is calculated as follows: for example, for $85 \%$ of BEP flow rate, the corresponding relative shaft power according to the manufacturer's $Q P$ curve is used to generate a flow rate estimate with the $n_{\mathrm{q}}$-based method's characteristic curve. The difference of the generated flow rate estimate and the flow rate $Q^{*}=85 \%$ is then expressed as a percentage of the BEP flow rate. As seen in Figs. 4 and 6 , the difference between the characteristic curves 
Fig. 4 Characteristic shaft power vs. flow rate curves created with the presented method compared with manufacturers' corresponding curves (Apex Fluid Engineering Ltd, "TD EndSuction Pumps 2003; Sulzer Pumps Finland Oy n.d.; Sulzer Pumps Finland Oy 2005) and measured curves



based on Gülich's set of curves and the manufacturers' curves falls within a range of -5 to $21 \%$ of $Q_{\mathrm{BEP}}$. When Stepanoff's curves are used, the corresponding difference ranges from -7 to $6 \%$ of $Q_{\mathrm{BEP}}$.

\section{$Q H$-curve-based flow rate estimation}

The operation of the presented method when head vs. flow rate curves are used was evaluated with the same analysis procedure as in the previous sub-section. Figure 7 presents the relative $Q H$ curves of the studied pumps.

As seen in Fig. 7, the shut-off head of the Gülichbased curves is significantly higher than that of the manufacturers' and the measured curves. Furthermore, Stepanoff-based curves of lower specific speeds can produce more than one flow rate estimate with a certain head value, which can limit their usability at low flow rates. The global maximum present in Stepanoff-based curves of low $n_{\mathrm{q}}$ can also lead to a flow rate value being unavailable for values of head that the pump may produce. This can be seen in Fig. 7: head values of $H^{*}>$ $110 \%$, which can be found on the measured curves, do not yield flow rate estimates when using the Stepanoffbased curves.
Figure 8 presents the measured estimation error for the curves when head is used to derive flow rate estimates. The error is calculated as described in the previous sub-section. Due to the global maximum of the Stepanoff-based curve, no difference to the measured curve could be calculated at $60 \%$ for the first pump and at 60 and $70 \%$ of $Q_{\mathrm{BEP}}$ for the second pump shown in Fig. 8. Between 85 and $120 \%$ of $Q_{\mathrm{BEP}}$, the estimation error of the Gülich-based curves is within -7 to $8 \%$ of $Q_{\mathrm{BEP}}$, while for the Stepanoff-based estimator it is within -10 to $7 \%$ of $Q_{\text {BEP. }}$ The results show that the demonstrated accuracy of the presented method, also with the $Q H$ method approach, is sufficient for detecting if a pump is running outside its recommended operating range.

In Fig. 9, a comparison of the flow rate estimates provided by the basic $Q H$ method and the $n_{\mathrm{q}}$-based method is presented. The difference is calculated as described in the previous sub-section, where the $Q P$ method was used as the basis of the comparison. The Gülich-based curves can be seen to differ from the manufacturers' curves by 10 to $21 \%$ of $Q_{\mathrm{BEP}}$ at flow rates between 60 and $70 \%$ of $Q_{\mathrm{BEP}}$. At flow rates from 85 to $120 \%$ of $Q_{\mathrm{BEB}}$, the difference is within -5 to $7 \%$ of $Q_{\mathrm{BEP}}$. The Stepanoff-based curves correspond with the 

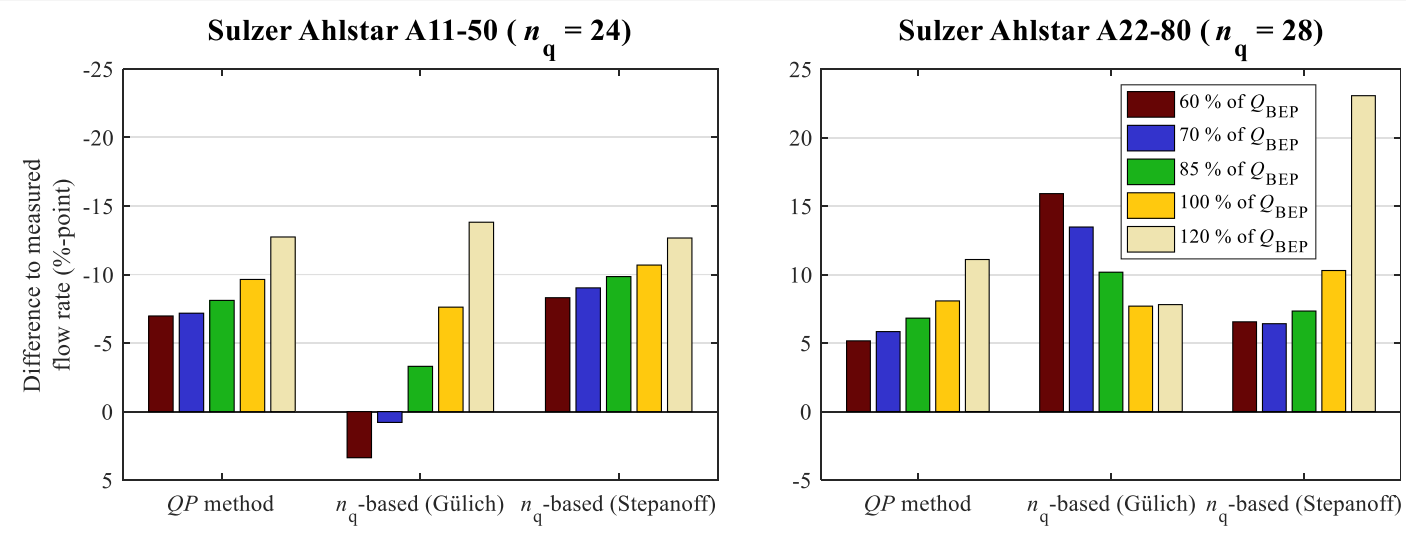

Fig. 5 The errors of the basic $Q P$ estimation method and the presented estimation method with respect to the measured values expressed as a percentage of the BEP flow rate. For ease of comparison, the vertical axis in the graph for Sulzer Ahlstar A11-50 has been inverted

manufacturers' curves more closely. With the exception of flow rates from 60 to $70 \%$ of $Q_{\mathrm{BEP}}$ with Sulzer Ahlstar A22-80, where the global maximum head value limits the usability of the curves for flow rate estimation, the difference between Stepanoff-based and manufacturers' curves is within -2 to $4 \%$ of $Q_{\mathrm{BEP}}$.

\section{Conclusion}

The monitoring and control of large populations of pumps could be conducted cost-efficiently through the use of an Internet connection and an easy-toemploy flow rate estimation method, which employs the nominal operating values of the pumps and estimates or measurements of their shaft powers or

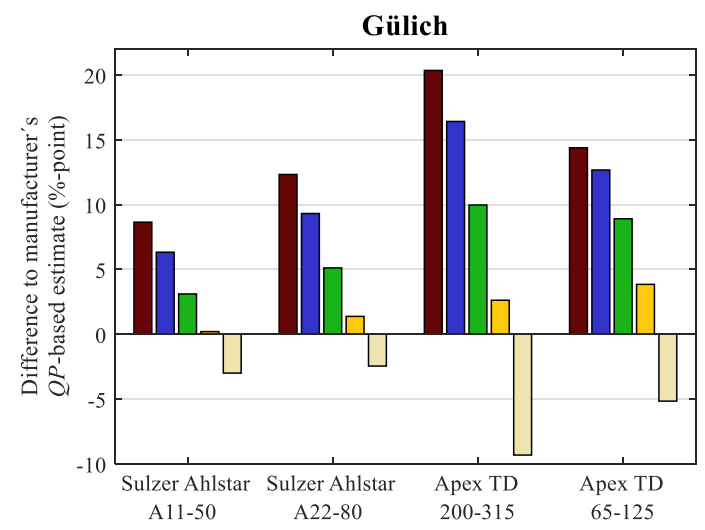

Fig. 6 Difference between the estimates provided by the presented method and the manufacturer's $Q P$ curve expressed as a percentage of the BEP flow rate. For $120 \%$ of BEP flow rate, due to produced heads. Currently existing flow rate estimation methods can be cumbersome to use due to the amount of data required to execute them. Also, many of the methods employ a variable-speed drive to estimate the flow rate, while the majority of pumps are actually fixed-speed.

In this paper, a novel fast-to-employ flow rate estimator for centrifugal pumps was presented. The presented estimator requires the input of a pump's nominal operating parameters, which makes it easier to employ than other so far developed estimators. Its performance was evaluated by comparing its operation and accuracy to the already existing estimation methods, where a characteristic $Q P$ or $Q H$ curve provided by a pump manufacturer is used to provide estimates for flow rate. The presented estimation

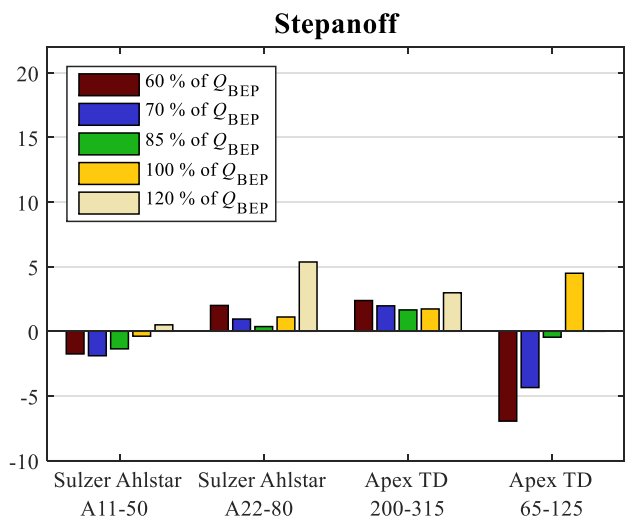

the shapes of the curves for APEX TD 65-125, no difference could be calculated between the flow rates indicated by the published curve and the presented Stepanoff-based estimation method 

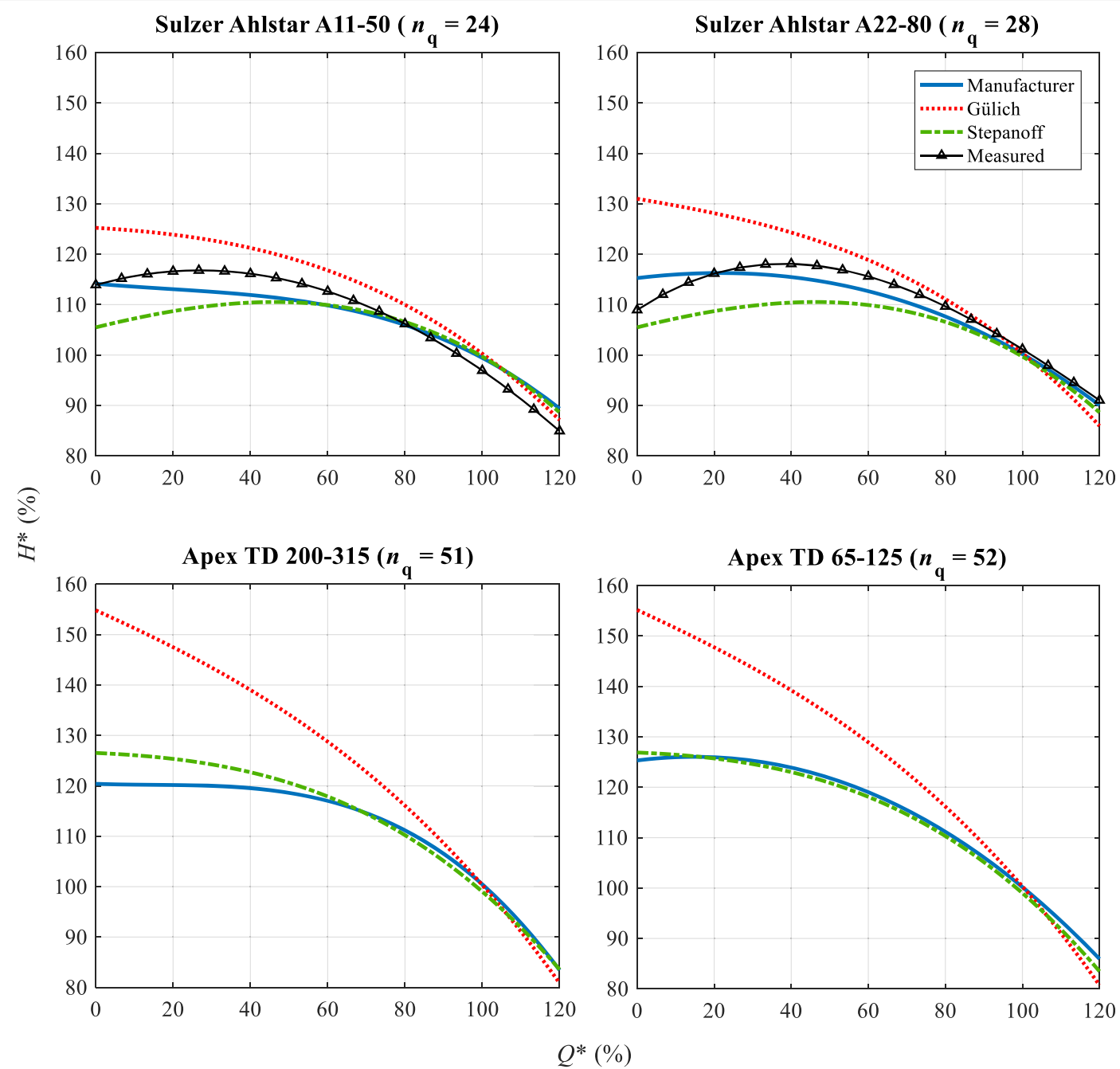

Fig. 7 Characteristic head vs. flow rate curves created with the presented method compared with manufacturers' corresponding curves (Apex Fluid Engineering Ltd, “TD End-Suction Pumps 2003; Sulzer Pumps Finland Oy n.d.; Sulzer Pumps Finland Oy 2005) and measured curves
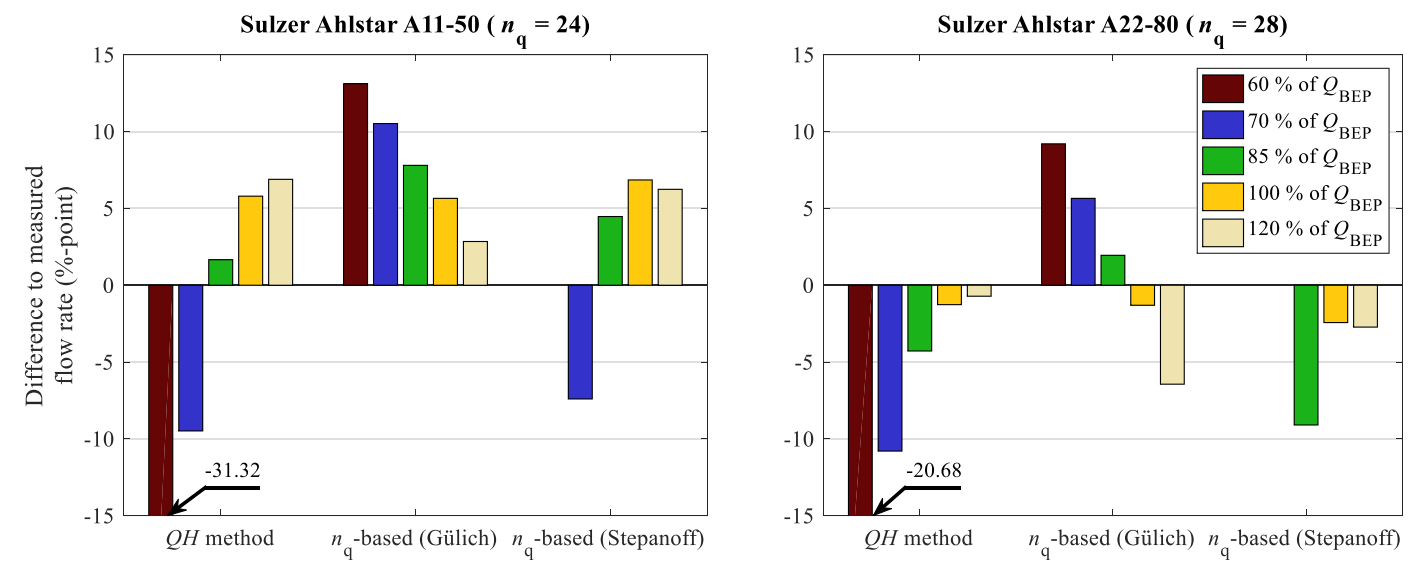

Fig. 8 The errors of the basic $Q H$ estimation method and the presented estimation method with respect to the measured values expressed as a percentage of the BEP flow rate 


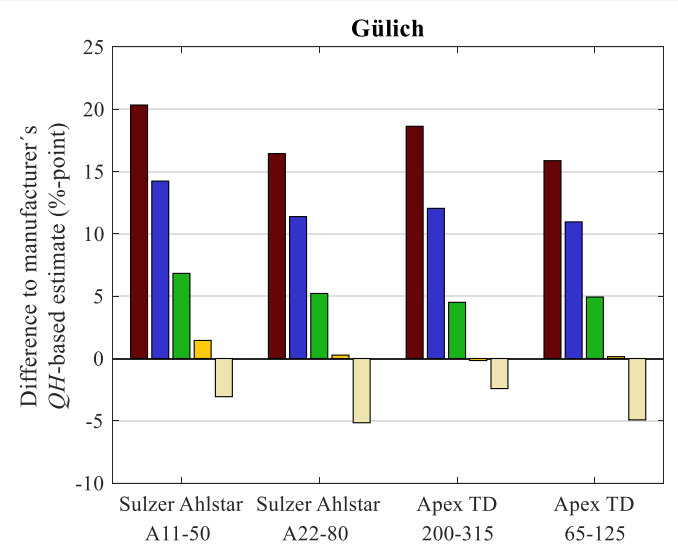

Fig. 9 Difference between the estimates provided by the presented method and the manufacturer's $Q H$ curve expressed as a percentage of $Q_{\mathrm{BEP}}$. For $60 \%$ of $Q_{\mathrm{BEP}}$, due to the shapes of the

method is based on sets of relative $Q P$ and $Q H$ curves, in which the curves' shapes are defined as a function of the pump specific speed. In this study, the curve sets published by Gülich (Gülich 2014) and Stepanoff (Stepanoff 1957) were used to generate the $Q P$ and $Q H$ curves of four pumps, and the accuracy of flow rate estimation based on these curves was evaluated.

The $Q P$ and $Q H$ curves of the studied pumps were generated with the presented estimation method and compared with the corresponding curves provided by the pumps' manufacturers. With flow rates other than at the immediate vicinity of the BEP flow rate, the $Q P$ curves generated with the presented method significantly deviated from the manufacturer's curves when using Gülich's work as the source for the $n_{\mathrm{q}}$-based relative characteristic curves. When Stepanoff's work was used as the source, the estimated characteristic curves followed the manufacturer's published curves more closely, their difference staying within $\pm 7 \%$ of BEP flow rate. Also out of the generated $Q H$ curves, Stepanoff-based curves corresponded better with the manufacturers' curves. At low specific speeds, however, a global maximum head value may occur on the Stepanoff-based $Q H$ curves, which can limit their usability at low flow rates.

The actual $Q P$ and $Q H$ curves of two pumps were measured in a laboratory pumping system. The accuracy of the basic $Q P$ and $Q H$-curve based estimation method and that of the presented estimation method was evaluated based on the measured curves. The flow rate estimator based on Stepanoff's set of relative $Q P$ curves deviated from the measured curves approximately as greatly as the basic $Q P$-based estimation method did.

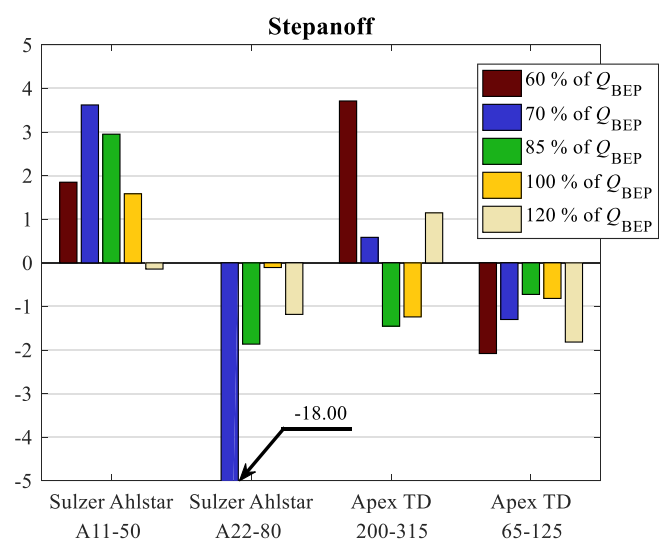

curves for Sulzer Ahlstar A22-80, no difference could be calculated between the flow rates indicated by the published curve and the presented Stepanoff-based estimation method

The error of the Stepanoff-based method was 7 to $15 \%$ of the BEP flow rate through a flow rate range of 60 to $100 \%$ of the BEP flow rate. In $Q H$-curve-based estimation, the presented method, although not always usable at low flow rates with low specific speed pumps, shows accuracy similar to the basic $Q H$ method.

The accuracy of the method can be expected to be limited by the deviation in the performance of pumps of equal specific speeds and by the performance changes that pumps go through during their lifetimes, such as impeller wear and fouling. As such, the accuracy of the presented estimator cannot rival that of an actual flow measurement. However, the experimental evaluation and the simulation suggest that the accuracy of the estimator is sufficient to highlight inefficiently running centrifugal pumps from large pump populations. Furthermore, the accuracy of the estimator could be improved by using more suitable and modern sets of relative $n_{\mathrm{q}}$-based curves when they become available.

Acknowledgements Open access funding provided by Lappeenranta University of Technology (LUT).

Funding information This work was carried out in the Efficient Energy Use (EFEU) research program coordinated by CLIC Innovation Ltd. with funding from the Finnish Funding Agency for Technology and Innovation, Tekes (which has since then been merged into Business Finland Ltd.).

\section{Compliance with ethical standards}

Conflict of interest The authors declare that they have no conflict of interest. 
Appendix

Table 3 The coefficients of the third-degree polynomial curves fitted on the digitized values and for the measured $Q P$ curves

\begin{tabular}{|c|c|c|c|c|c|}
\hline Source & $n_{\mathrm{q}}$ & $c_{1}$ & $c_{2}$ & $c_{3}$ & $c_{4}$ \\
\hline \multirow[t]{5}{*}{ Gülich } & 20 & $-4.988 \times 10^{-5}$ & $1.124 \times 10^{-2}$ & $-1.728 \times 10^{-3}$ & 36.822 \\
\hline & 50 & $-3.692 \times 10^{-5}$ & $9.139 \times 10^{-3}$ & $-7.409 \times 10^{-2}$ & 51.151 \\
\hline & 100 & $-2.549 \times 10^{-5}$ & $8.303 \times 10^{-3}$ & $-4.710 \times 10^{-1}$ & 90.243 \\
\hline & 150 & $-1.500 \times 10^{-4}$ & $3.362 \times 10^{-2}$ & -2.489 & $1.614 \times 10^{2}$ \\
\hline & 250 & $-2.931 \times 10^{-4}$ & $6.391 \times 10^{-2}$ & -5.352 & $2.889 \times 10^{2}$ \\
\hline \multirow[t]{7}{*}{ Stepanoff } & 17 & $4.423 \times 10^{-6}$ & $-1.146 \times 10^{-3}$ & $7.463 \times 10^{-1}$ & 31.957 \\
\hline & 29 & $-1.362 \times 10^{-5}$ & $1.540 \times 10^{-3}$ & $5.954 \times 10^{-1}$ & 38.014 \\
\hline & 43 & $-1.857 \times 10^{-5}$ & $2.768 \times 10^{-3}$ & $3.999 \times 10^{-1}$ & 49.762 \\
\hline & 58 & $-7.106 \times 10^{-6}$ & $-1.342 \times 10^{-4}$ & $4.650 \times 10^{-1}$ & 61.831 \\
\hline & 77 & $-1.346 \times 10^{-5}$ & $2.260 \times 10^{-3}$ & $-8.097 \times 10^{-3}$ & 91.058 \\
\hline & 110 & $-7.226 \times 10^{-5}$ & $1.946 \times 10^{-2}$ & -2.141 & $1.907 \times 10^{2}$ \\
\hline & 178 & $-2.428 \times 10^{-5}$ & $7.058 \times 10^{-3}$ & -2.287 & $2.805 \times 10^{2}$ \\
\hline Measured Sulzer A11-50 & 24 & $-1.637 \times 10^{-5}$ & $3.310 \times 10^{-3}$ & 0.379 & 38.572 \\
\hline Measured Sulzer A22-80 & 28 & $-1.082 \times 10^{-5}$ & $2.418 \times 10^{-3}$ & 0.423 & 48.553 \\
\hline
\end{tabular}

Table 4 The coefficients of the third degree polynomial curves fitted on the digitized values and for the measured $Q H$ curves

\begin{tabular}{|c|c|c|c|c|c|}
\hline Source & $n_{\mathrm{q}}$ & $c_{1}$ & $c_{2}$ & $c_{3}$ & $c_{4}$ \\
\hline \multirow[t]{9}{*}{ Gülich } & 20 & $-8.987 \times 10^{-6}$ & $-1.385 \times 10^{-3}$ & $2.372 \times 10^{-2}$ & $1.207 \times 10^{2}$ \\
\hline & 30 & $-1.503 \times 10^{-5}$ & $-1.547 \times 10^{-4}$ & $-1.59 \times 10^{-1}$ & $1.329 \times 10^{2}$ \\
\hline & 40 & $-1.996 \times 10^{-5}$ & $1.044 \times 10^{-3}$ & $-3.495 \times 10^{-1}$ & $1.447 \times 10^{2}$ \\
\hline & 50 & $-1.207 \times 10^{-5}$ & $-8.218 \times 10^{-4}$ & $-3.305 \times 10^{-1}$ & $1.536 \times 10^{2}$ \\
\hline & 60 & $-2.043 \times 10^{-5}$ & $4.232 \times 10^{-4}$ & $-4.500 \times 10^{-1}$ & $1.616 \times 10^{2}$ \\
\hline & 70 & $-2.936 \times 10^{-5}$ & $1.587 \times 10^{-3}$ & $-5.343 \times 10^{-1}$ & $1.677 \times 10^{2}$ \\
\hline & 80 & $-3.761 \times 10^{-5}$ & $2.508 \times 10^{-3}$ & $-5.921 \times 10^{-1}$ & $1.728 \times 10^{2}$ \\
\hline & 100 & $-7.165 \times 10^{-5}$ & $7.739 \times 10^{-3}$ & $-8.479 \times 10^{-1}$ & $1.808 \times 10^{2}$ \\
\hline & 250 & $-2.650 \times 10^{-4}$ & $5.307 \times 10^{-2}$ & -5.052 & $3.402 \times 10^{2}$ \\
\hline \multirow[t]{7}{*}{ Stepanoff } & 17 & $-1.401 \times 10^{-5}$ & $-1.052 \times 10^{-3}$ & $1.873 \times 10^{-1}$ & $1.055 \times 10^{2}$ \\
\hline & 29 & $-1.709 \times 10^{-5}$ & $-3.945 \times 10^{-4}$ & $9.690 \times 10^{-2}$ & $1.108 \times 10^{2}$ \\
\hline & 43 & $-2.402 \times 10^{-5}$ & $1.133 \times 10^{-3}$ & $-7.656 \times 10^{-2}$ & $1.195 \times 10^{2}$ \\
\hline & 58 & $-7.002 \times 10^{-6}$ & $-2.597 \times 10^{-3}$ & $-5.300 \times 10^{-4}$ & $1.319 \times 10^{2}$ \\
\hline & 77 & $-1.269 \times 10^{-5}$ & $-2.290 \times 10^{-3}$ & $-1.772 \times 10^{-1}$ & $1.531 \times 10^{2}$ \\
\hline & 110 & $-3.320 \times 10^{-5}$ & $3.520 \times 10^{-3}$ & -1.030 & $1.997 \times 10^{2}$ \\
\hline & 178 & $-9.731 \times 10^{-6}$ & $3.283 \times 10^{-3}$ & -2.185 & $2.968 \times 10^{2}$ \\
\hline Measured Sulzer A11-50 & 24 & $2.200 \times 10^{-6}$ & $-4.062 \times 10^{-3}$ & $2.142 \times 10^{-1}$ & $1.139 \times 10^{2}$ \\
\hline Measured Sulzer A22-80 & 28 & $1.923 \times 10^{-5}$ & $-7.801 \times 10^{-3}$ & $5.099 \times 10^{-1}$ & $1.089 \times 10^{2}$ \\
\hline
\end{tabular}


Open Access This article is distributed under the terms of the Creative Commons Attribution 4.0 International License (http:// creativecommons.org/licenses/by/4.0/), which permits unrestricted use, distribution, and reproduction in any medium, provided you give appropriate credit to the original author(s) and the source, provide a link to the Creative Commons license, and indicate if changes were made.

\section{References}

Ahonen, T., Tamminen, J., Ahola, J., Viholainen, J., Aranto, N., \& Kestilä, J. (2010). Estimation of pump operational state with model-based methods. Energy Conversion and Management, 51(6), 1319-1325.

Ahonen T., Tamminen J., Ahola J. and Niemelä M., (2011). "Accuracy study of frequency converter estimates used in the sensorless diagnostics of induction-motor-driven systems," in Proceedings of the 14th European Conference on Power Electronics and Applications, Birmingham

Ahonen, T., Tamminen, J., Ahola, J., \& Kestilä, J. (2012a). Frequency-converter-based hybrid estimation method for the pump operational state. IEEE Transactions on Industrial Electronics, 59(12), 4803-4809.

Ahonen, T., Kortelainen, J., Tamminen, J., \& Ahola, J. (2012b). Centrifugal pump operation monitoring with motor phase current measurement. Electrical Power and Energy Systems, 42, 188-195.

de Almeida A., Fong J. and Falkner H., (2015). "New European ecodesign regulation for electric motors and drives," in 9th International Conference on Energy Efficiency in Motor Driven Systems - EEMODS 2015, Helsinki

Apex Fluid Engineering Ltd, "TD End-Suction Pumps," (2003). [Online]. Available: http://www.apexpumps. com/assets/downloads/TDtechnicalbooklet.pdf. [Accessed 105 2017].

Backlund, S., \& Thollander, P. (2015). Impact after three years of the Swedish energy audit program. Energy, 82, 54-60.

European Commission. (2012). "Directive 2012/27/EU of the European Parliament and of the Council of 25 October 2012 on energy efficiency, amending Directives 2009/125/ EC and 2010/30/EU and repealing Directives 2004/8/EC and 2006/32/EC," Official Journal of the European Union

European Commission. (2014). Communication from the Commission to the European Parliament and the Council: Energy efficiency and its contribution to energy security and the 2030 framework for climate and energy policy. Brussels, 23,7 .

Fleiter, T., \& Eichhammer, W. (2012). Energy efficiency in electric motor systems: Technology, saving potentials and policy options for developing countries. Vienna: United Nations Industrial Development Organization.

Gülich J.. (2014) Centrifugal pumps, third ed., Springer.

Kaya, D., Yagmur, E., Yigit, K., Kilic, F., Eren, A., \& Celik, C. (2008). Energy efficiency in pumps. Energy Conversion and Management, 49, 1662-1673.

Kernan D., Sabini E., Ganzon N. and Stavale A., (2007). "Method for determining pump flow without the use of traditional sensors," US Patent 2007/0212210 A1

Leonow S. and Mönnigmann M.. (2013) "Soft sensor based dynamic flow rate estimation in low speed radial pumps," in Proceedings of 2013 European control conference ECC13, Zürich

Mamade A., Sousa C., Marques A., Loureiro D., Alegre H. and Covas D.. (2015) "Energy auditing as a tool for outlining major inefficiencies: Results from a real water supply system, " in Procedia Engineering, 119, 1098, 1108.

Nash, J. (1997). Direct torque control, induction motor vector control without an encoder. IEEE Transactions on Industry Applications, 33(2), 333-341.

Nesbitt B., (2006) Handbook of pumps and pumping, First ed., Elsevier, Roles \& Associates Ltd

Price L. and Lu H.. (2011) "Industrial energy auditing and assessments: a survey of programs around the world," in eceee Summer Study - energy efficiency first: The foundation of a low-carbon society, France, 2011.

Saidur, R. (2010). A review on electrical motors energy use and energy savings. Renewable and Sustainable Energy Reviews, 14, 877-898.

Stepanoff, A. (1957). Centrifugal and axial flow pumps (Second ed.). Malabar, FL: Krieger Publishing.

Sulzer Pumps Finland Oy, Ahlstar UP A22-80 (125-80-250). Characteristic curve K50659., Mänttä: Sulzer Pumps Finland Oy, 2005.

Sulzer Pumps Finland Oy, Ahlstar A11-50 (80-50-200). Characteristic curve K18710, Sulzer Pumps Finland Oy.

Sulzer Pumps Ltd., Centrifugal pump handbook. (1998), 2nd ed., Winterthur, Switzerland: Elsevier.

Tamminen, J., Viholainen, J., Ahonen, T., Ahola, J., Hammo, S., \& Vakkilainen, E. (2014). Comparison of model-based flow rate estimation methods in frequency-converter-driven pumps and fans. Energy Efficiency, 7(3), 493-505.

Tinazzi, F., \& Zigliotto, M. (2015). Torque estimation in highEfficency IPM synchronous motor drives. IEEE Transactions on Energy Conversion, 30(3), 983-990.

United States Department of Energy. (1998). United States industrial electric motor systems market opportunities assessment. Burlington, MA: Xenergy, Inc.

Volk, M. (2005). Pump characteristics and applications (2nd ed.). Florida: CRC Press.

Waide P. and Brunner C.. (2011) "Energy-efficiency policy opportunities for electric motor-driven systems," International Energy Agency 